\title{
Can We Rely on Susceptibility-Weighted Imaging for Subthalamic Nucleus Identification in Deep Brain Stimulation Surgery?
}

\author{
Maarten Bot, MD* \\ Lo Bour, PhD $\ddagger$ \\ Rob M. de Bie, MD, PhD \\ Maria Fiorella Contarino, MD, \\ $\mathrm{PhD} \ddagger \S$ \\ P. Richard Schuurman, MD, \\ PhD*
}

Pepijn van den Munckhof, MD, PhD*

Departments of *Neurosurgery and $\ddagger$ Neurology and Clinical Neurophysiology, Academic Medical Center, Amsterdam, the Netherlands; §Haga Teaching Hospital, Den Haag, the Netherlands

\section{Correspondence:}

Maarten Bot, MD,

Department of Neurosurgery,

Academic Medical Center,

Meibergdreef 9,

1105 AZ Amsterdam,

Netherlands.

E-mail: m.bot@amc.nl

Received, March 8, 2015.

Accepted, October 4, 2015.

Published Online, November 19, 2015.

Copyright $\odot 2015$ by the

Congress of Neurological Surgeons.

BACKGROUND: Susceptibility-weighted imaging (SWI) offers significantly improved visibility of the subthalamic nucleus (STN) compared with traditional T2-weighted imaging. However, it is unknown whether the representation of the nucleus on SWI corresponds to the neurophysiological location of the STN.

OBJECTIVE: To determine the correlation between the intraoperative electrophysiological activity of the STN and the representation of the nucleus on different magnetic resonance imaging (MRI) sequences used for deep brain stimulation target planning. METHODS: At stereotactic target depth, microelectrode recordings (MERs) of typical STN neuronal activity were mapped on 3 different preoperative MRI sequences: 1.5-T SWI, 1.5-T T2-weighted, and 3-T T2-weighted MRI. For each MRI sequence, it was determined whether the MER signal was situated inside or outside the contour of the STN.

RESULTS: A total of 196 MER tracks in 34 patients were evaluated. In 165 tracks (84\%), typical electrophysiological STN activity was measured. MER activity was situated more consistently inside hypointense STN contour representation on 1.5- and 3-T T2weighted images compared with SWI (99\% and $100 \%$ vs $79 \%$, respectively). The $21 \%$ incongruence of electrophysiological STN activity outside the STN contour on SWI was seen almost exclusively in the anterior and lateral microelectrode channels.

CONCLUSION: STN representation on SWI does not correspond to electrophysiological STN borders. SWI does not correctly display the lateral part of the STN. When aiming to target the superolateral sensorimotor part of the STN during deep brain stimulation surgery, SWI does not offer an advantage but a disadvantage compared with conventional T2. Future research is needed to determine whether these findings may also apply for high-field SWI.

KEY WORDS: Deep brain stimulation, Parkinson disease, STN, SWI, Target planning

Neurosurgery 78:353-360, 2016

DOI: $10.1227 /$ NEU. 0000000000001130

www.neurosurgery-online.com

B ilateral deep brain stimulation (DBS) of the subthalamic nucleus (STN) is the most effective surgical procedure for patients with advanced Parkinson disease. ${ }^{1,2}$ The precise identification of the STN target is paramount for maximizing therapeutic benefits while minimizing side effects such as muscle contraction, dizziness, mood changes, and akinesias, which could occur when the electrode is placed inappropriately. ${ }^{3}$ Preoperative visualization of the STN on T2-weighted magnetic resonance imaging

ABBREVIATIONS: DBS, deep brain stimulation; MER, microelectrode recording; STN, subthalamic nucleus; SWI, susceptibility-weighted imaging
(MRI) enables direct targeting based on a patient's own brain anatomy. 4,5

Notwithstanding the benefits of T2-weighted MRI, it remains challenging to directly visualize the STN. ${ }^{6}$ It is frequently difficult to distinguish the STN from the ventromedially located substantia nigra and from the anterodorsally located pallidofugal fiber pathways, which are also hypointense on T2-weighted sequences. ${ }^{7,8}$ Various alternative MRI sequences and techniques have been suggested to offer improved visibility of the STN. Susceptibility-weighted imaging (SWI) is currently considered to offer significantly improved visibility of iron-dense structures such as the STN. ${ }^{9,10}$ Moreover, SWI enhances the precise visualization of deep 
cerebral veins and tiny parenchymal vessels, thereby facilitating the planning of electrode trajectories through the brain with a lower risk of intracranial hemorrhage. ${ }^{11,12}$

In most centers, stereotactic visualization of the STN is the first step in an operative procedure in awake patients that successively includes neurophysiological mapping of the target region through microelectrode recordings (MERs) and intraoperative test stimulation to identify the optimal clinical target. ${ }^{13,14}$ With recent improvements in preoperative STN visualization on stereotactic MRI, several groups have proposed omitting MER or test stimulation and relying solely on MRI for determination of optimal lead placement in the STN ${ }^{15-18}$ It is evident that proper visualization of the STN is of crucial importance for this strategy. Although SWI is considered to improve visibility of the STN and therefore is another possible radiological refinement in direct stereotactic targeting, it had not yet been evaluated in DBS surgery. To address this issue, we compared STN representation on stereotactic MRI sequences, including SWI, with electrophysiological STN borders as determined by MER.

\section{METHODS}

\section{Patients}

Information was collected from all patients who underwent MERguided DBS implantation for Parkinson disease in our hospital between March 2012 and March 2014 for whom a complete set of SWI and T2 and 3-dimensional T1 MRI was available. We began obtaining the SWI systematically in all patients beginning in January 2013 in an attempt to improve visualization of the STN during clinical practice.

\section{Surgical Procedure}

Three to 6 months before the day of surgery, patients underwent a 3-T nonstereotactic MRI (Siemens, Malvern, Pennsylvania). On the day of surgery, the Leksell stereotactic G frame (Elekta AB, Stockholm, Sweden) was placed, and patients underwent a preoperative frame-based 1.5-T stereotactic MRI (Siemens) using axial and coronal T2-weighted, 1.5-T axial SWI, and post-gadolinium volumetric axial T1-weighted sequences. Axial T2 and SWI sequences were obtained in a slab covering the brain from the superior cerebellar peduncle to the top of the lateral ventricles. The coronal T2 MRI sequence covered an area from the anterior tips of the frontal to the occipital horns. Preoperative 3-T MRI sequences were coregistered with stereotactic volumetric T1-weighted sequence with the use of SurgiPLan (Elekta AB). STN target planning was started with standard stereotactic coordinates relative to the midcommissural point: $11 \mathrm{~mm}$ in the lateral direction, $2 \mathrm{~mm}$ in the posterior direction, and $4 \mathrm{~mm}$ in the ventral direction. Target planning was subsequently optimized, ie, direct target planning based on red nucleus and STN representation on 1.5- and 3-T axial and coronal T2 sequences. Trajectory planning was subsequently done with a postgadolinium volumetric axial T1-weighted sequence. Entry points were chosen precoronal and 3 to $4 \mathrm{~cm}$ lateral from the midline on a suitable gyrus. Planned trajectories were inspected to avoid penetration of ventricles, caudate nucleus, and blood vessels. SWI was not used for targeting or evaluation of (venous) vessel penetration. All patients were operated on under local anesthesia with the head frame secured to the operation table. Patients were placed in supine position with the head elevated $20^{\circ}$ to $30^{\circ}$ to minimize the outflow of cerebrospinal fluid through the burr hole. Cerebrospinal fluid outflow was further minimized by applying fibrin glue after microelectrode positions were in place to begin MER. In bilateral cases, surgery started on the side of the brain contralateral to the most affected side of the body. It was noted when adjustments in the ring and arc were needed after burr hole placement for optimizing the entrance of the microelectrodes. Two to 4 microelectrodes (FHC, Bowdoin, Maine) were inserted through a 12-mm-diameter burr hole. Multitract MER (Leadpoint; Medtronic Inc, Minneapolis, Minnesota) started 6 or $5 \mathrm{~mm}$ above the target point, and the microelectrodes were manually advanced with $0.5-\mathrm{mm}$ steps. In most cases, we used 3-channel recordings with the planned trajectory as the central channel, with additional lateral and anterior channels at a 2 -mm distance from the central channel. ${ }^{19}$ Recordings were extended 2 to $3 \mathrm{~mm}$ beyond the target point until electrophysiological STN signal stopped or substantia nigra pars reticulata signal was measured. Therapeutic effects and possible side effects were evaluated by monopolar electric stimulation through the macrotip of the microelectrode at 2 to 3 different depths along the trajectories with positive electrophysiological STN signal. After the optimal MER pass was determined, the DBS lead (model 3389; Medtronic Inc) was implanted. The DBS electrode was secured to the skull with Stimloc system (Medtronic Inc). Adequate lead placement was evaluated with fluoroscopy. Subsequent implantation of 1 or 2 pulse generators was done in a subcutaneous or subpectoral pocket in the infraclavicular region under general anesthesia on the same day. On the postoperative day, a computed tomography (CT) scan was made to evaluate lead position, to assess the postoperative pneumocephalus, and to rule out asymptomatic hemorrhage.

\section{MRI Parameters for Each Sequence}

For 1.5-T SWI, the parameters were as follows: repetition time, 49 milliseconds; echo time, 40 milliseconds; field of view, $250 \times 250 \mathrm{~mm}$; voxel size, $1 \times 1 \times 1 \mathrm{~mm}$; slice thickness, $1.0 \mathrm{~mm}$; and acquisition time, 6:43 minutes. For 1.5-T T2, the parameters included the following: repetition time, 5750 milliseconds; echo time, 99 milliseconds; field of view, $256 \times 256 \mathrm{~mm}$; voxel size, $1 \times 1 \times 2 \mathrm{~mm}$; slice thickness, $2.0 \mathrm{~mm}$; and acquisition time, 4:43 minutes. For 3.0-T T2, the parameters included the following: repetition time, 4625 milliseconds; echo time, 80 milliseconds; field of view, $230 \times 200 \mathrm{~mm}$; voxel size, $0.5 \times 0.5 \times 2 \mathrm{~mm}$; slice thickness, $2.0 \mathrm{~mm}$; and acquisition time, $4: 46$ minutes.

\section{Evaluation of Electrophysiologically and MRI- Defined STN}

Electrophysiological STN activity was considered to be present when a broadening of background noise and tonic and irregular discharge pattern with occasional bursts were detected during MER. During surgery, electrophysiological recordings were reviewed and interpreted by the neurophysiologist (L.B.), neurologist (R.d.B. or M.F.C.), and DBS surgeon (R.S. or P.v.d.M.). Postoperatively, electrophysiological recordings were reviewed and double-checked for the presence of typical STN activity on target depth by the first and last authors. All MERs were categorized into 1 of 3 groups: MER with STN activity, MER without STN activity, and MER with activity that could indicate STN activity but lacking 1 or several of its typical features. MER trajectories were evaluated relative to STN representation on 1.5-T T2 MRI, 3-T T2 MRI, and 1.5-T SWI. The location of trajectories in relation to STN representation at target depth was visually evaluated on axial slices for all sequences. On both T2 sequences, the STN contour representation was considered to be lateral to the anterior border of the red nucleus with hypointense head and 
thinning posterior tail. On the SWI sequence, the STN contour was considered to be represented as a hypointense structure in mainly uniform hyperintense surrounding white matter lateral to the anterior margin of the (hypointense) red nucleus (Figure 1). ${ }^{10}$ The projection of every single MER on each MRI sequence was categorized into 1 of 3 groups: inside STN representation, outside STN representation, and at the border of STN representation. We then compared the delineation of the STN as defined by MER, which we took as golden standard, and as outlined by various MRI sequences and calculated the sensitivity and specificity for all 3 sequences.

The accuracy and precision of stereotactic methods were checked by comparing the intended electrode localization (based on intraoperative MER and clinical testing) to the final electrode position on postoperative CT coregistered to stereotactic MRI. For this, the $x$ and $y$ coordinates of final lead at target depth were compared with intraoperative coordinates. Both absolute and euclidean errors were calculated. Mean values are presented as $\pm \mathrm{SD}$.

\section{RESULTS}

A total of 66 DBS leads were placed and 196 microelectrode tracks were performed in 34 patients with Parkinson disease. There were 22 male and 12 female patients. Average age at surgery was 61 years, with average disease duration of 11 years. Thirty-two patients underwent bilateral DBS placement. Two patients underwent unilateral surgery. Direct planning of the target point based on STN representation on T2-weighted sequences resulted in mean stereotactic coordinates of $10.4 \pm 0.8 \mathrm{~mm}$ lateral, $2.3 \pm$ $0.6 \mathrm{~mm}$ posterior, and $4.2 \pm 0.5 \mathrm{~mm}$ inferior relative to the midcommissural point. In the majority of patients, we noticed that the STN contour on SWI was thinner than on T2 and that the target point was localized relatively more lateral in the STN contour on SWI (Figure 1).

\section{Multielectrode Recording}

In 64 placements, MER was performed with 3 microelectrodes (central, anterior, and lateral channel). In 2 placements (in brain sides operated second), we decided to perform MER only in the central and anterior channel because previous MER and test stimulation on the lateral channel in the brain side operated first had shown minimal STN activity and low thresholds for internal capsule side effects. The total number of microelectrode tracks was 196. In 165 microelectrode tracks (84\%), typical electrophysiological STN activity was measured at target depth; in 15 microelectrode tracks (8\%), no electrophysiological STN activity was measured at target depth; and in 16 microelectrode tracks (8\%), border electrophysiological STN activity was measured at target depth.

\section{Correlation Between MER and MRI}

The results of MER and MRI categorization are presented in the Table. The percentage of microelectrodes measuring typical STN activity at target depth (central, anterior, and lateral channel combined) that were located inside or at the border of STNcontour representation was $99 \%$ for $1.5-\mathrm{T} \mathrm{T} 2,100 \%$ for 3-T T2, and only $79 \%$ for SWI. SWI incongruence (21\%) was seen almost exclusively in the anterior and lateral microelectrode channels. Figure 2 shows 2 examples of SWI incongruence.

\section{Correlation Between Final DBS Electrode Location and MRI Representation}

The final DBS electrode was placed in the central channel in 31 placements, in the anterior channel in 25 placements, and in the lateral channel in 8 placements. In 2 placements, we decided to

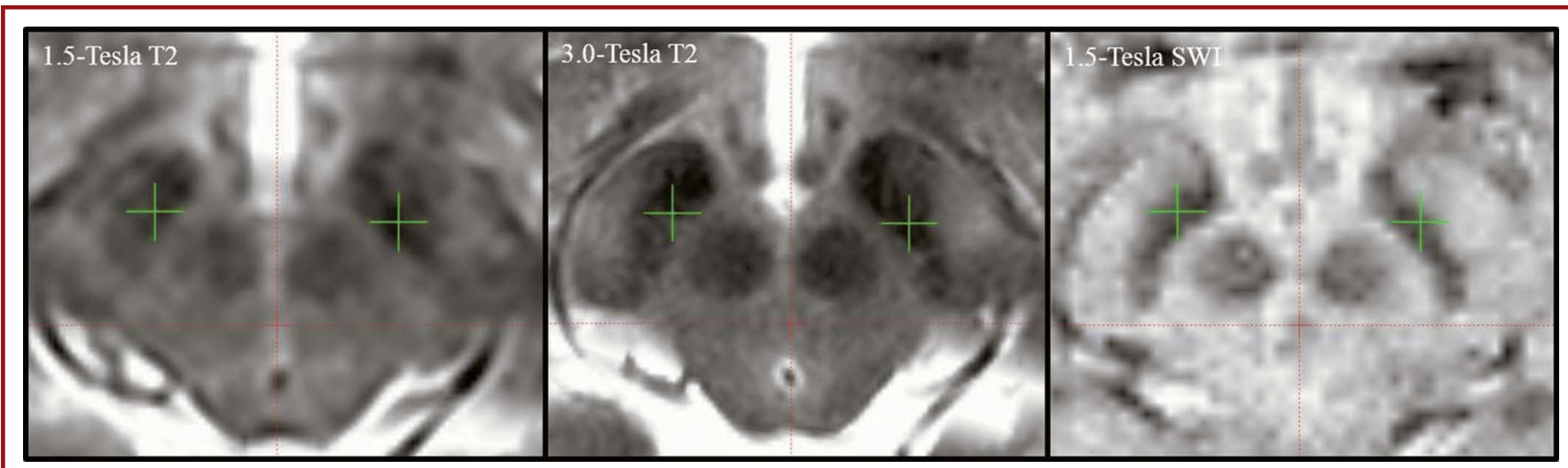

FIGURE 1. Axial midbrain section used for target planning during deep brain stimulation surgery displayed on 3 different magnetic resonance imaging (MRI) sequences. Left, a 1.5-T T2 sequence; middle, a 3.0-T T2 sequence; and right, a 1.5-T susceptibility-weighted imaging (SWI) sequence. The hypointense subthalamic nucleus (STN) signal is situated anterolateral to the red nucleus. On 3-T T2, both the red nucleus and the STN are better defined compared with the 1.5-T T2. STN representation on SWI is more clearly delineated compared with both T2 MRI sequences, with all surrounding structures almost uniformly hyperintense. The STN shape on SWI is a narrow, slightly curved hyperintense line, whereas on T2, it has a round head with decreasing hypointensity in posterior regions of its thinning tail. The green crosses represent the planned target point for both the left and right STN. Stereotactic coordinates of the center of the green crosses for the left were $10.5 \mathrm{~mm}$ lateral, $2.0 \mathrm{~mm}$ posterior, and $4.0 \mathrm{~mm}$ ventral. For the right, the stereotactic coordinates were $10.6 \mathrm{~mm}$ lateral, $1.4 \mathrm{~mm}$ posterior, and $4.0 \mathrm{~mm}$ ventral relative to the midcommissural point. The target point was localized relatively more lateral in the STN contour on SWI compared with both T2 sequences. 
TABLE. Overview of the Categorized Correlations Between Subthalamic Nucleus Microelectrode Recordings and Subthalamic Nucleus Representation on Different Magnetic Resonance Imaging Sequences ${ }^{a}$

\begin{tabular}{|c|c|c|c|c|c|c|c|c|c|c|}
\hline \multirow[b]{2}{*}{ MER } & \multirow[b]{2}{*}{ Channel } & \multicolumn{3}{|c|}{ 1.5T-T2 } & \multicolumn{3}{|c|}{ 3.0T-T2 } & \multicolumn{3}{|c|}{ 1.5T-SWI } \\
\hline & & In & Border & Out & In & Border & Out & In & Border & Out \\
\hline MER + & C & 58 & 0 & 0 & 58 & 0 & 0 & 53 & 1 & 4 \\
\hline MER + & L & 37 & 13 & 1 & 44 & 7 & 0 & 27 & 9 & 15 \\
\hline MER +/- & C & 4 & 0 & 0 & 4 & 0 & 0 & 4 & 0 & 0 \\
\hline $\mathrm{MER}+/-$ & A & 4 & 1 & 0 & 4 & 1 & 0 & 3 & 1 & 1 \\
\hline MER - & A & 3 & 0 & 2 & 5 & 0 & 0 & 5 & 0 & 0 \\
\hline MER - & L & 2 & 3 & 1 & 6 & 0 & 0 & 2 & 2 & 2 \\
\hline
\end{tabular}

${ }^{a}$ A, anterior channel; C, central channel; L, lateral channel; MER, multielectrode recording; SWI, susceptibility-weighted imaging; 1.5T-T2, 1.5-Tesla T2 weighted MRI; 3.0T-T2, 3.0-Tesla T2 weighted MRI. MER+ indicates microelectrode recordings positive for measuring typical electrophysiological subthalamic nucleus (STN) activity; MER+/-, microelectrode recordings with activity that could indicate STN activity but lacking 1 or several of its typical features; and MER-, microelectrode recordings without STN activity. Vertical columns indicate whether microelectrodes are situated inside (In), at the border (Border), or outside (Out) the STN representation on the respective magnetic resonance imaging (MRI) sequences. The horizontal rows indicate whether MER measured electrophysiological STN activity for each microelectrode channel. Both MER activity and STN representations on MRI are at target depth.

implant the final DBS electrode in the medial channel because of a suboptimal therapeutic window between the beneficial effects and side effects during test stimulation in the other channels. All final DBS electrode placements were located inside STN representation on 1.5- and 3-T T2 sequences. For SWI, however, 13 final DBS electrode placements (20\%) were outside STN representation. Again, this incongruence was seen mainly in the anterior (8 placements) and lateral (3 placements) channels.

\section{Accuracy and Precision of Stereotactic Methods}

The mean euclidean error between final electrode position and intended electrode position was $1.3 \pm 0.7 \mathrm{~mm}$, with mean $x$ and $y$ errors of $0.7 \pm 1.0 \mathrm{~mm}$ medial and $0.9 \pm 0.8 \mathrm{~mm}$ posterior, respectively.

\section{DISCUSSION}

\section{STN Representation on SWI}

We compared electrophysiological STN activity measured by MER with STN representation on different MRI sequences and found much lower correlation of MER STN activity with STN representation on the SWI sequence than on the 1.5- and 3-T T2 sequences, especially on the anterior and lateral microelectrode channels. Approximately one-third of anterior and lateral microelectrode channels with typical MER STN activity were located outside STN representation on SWI. SWI does not correctly display the lateral part of the STN. This finding is of outmost importance for STN target planning because the superolateral STN is thought to be the sensorimotor part of the nucleus ${ }^{20}$ and the optimal target for long-term stimulation. ${ }^{21,22}$ Indeed, onefifth of our current final DBS electrode placements were outside STN representation on SWI. SWI-based targeting of the STN therefore carries the risk of targeting the medial part of the nucleus, which is thought to comprise the limbic and associative territories. $^{20}$

In agreement with our results, other groups reported similarly high correspondence of electrophysiological STN activity with STN representation on both 1.5- and 3-T T2-weighted imaging. ${ }^{8,23-27}$ However, direct comparison between these studies is difficult because of the difference in methods used for comparing MER with MRI. Electrophysiological recordings were compared with 3-dimensional representation of STN, stereotactic coordinates of dorsal and ventral STN border on different orientations (axial, coronal, sagittal), stereotactic atlas STN representation, cadaveric STN representation, electrode contact position, or total electrode length of electrode track on postoperative imaging. Lefranc et $\mathrm{al}^{28}$ in 2014 compared the correspondence of electrophysiological STN activity with STN representation on 3-dimensional T2*-weighted angiography MRI in patients. Three-dimensional T2*-weighted angiography is assumed to be an equivalent susceptibility technique, although a comparison with SWI has never been performed. They concluded that there was a high visual correspondence between measured electrophysiological STN activity and MRI representation of the STN in the mediolateral axis without referring to their exact measurements. However, MER was performed under general anesthesia, which could make electrophysiological data difficult to interpret. Moreover, exact microelectrode setup and additional performed trajectories were not described, and a clear interrelationship between microelectrode channels and corresponding electrophysiological STN activity was not stated.

\section{Could SWI ReplaceT2?}

Better delineation of lateral STN borders would be desirable because they are known to be least clearly visible on T2 sequences. ${ }^{7}$ Several studies have propagated that SWI offers significantly improved visibility of STN borders. ${ }^{9,10,12,29,30}$ Our current 


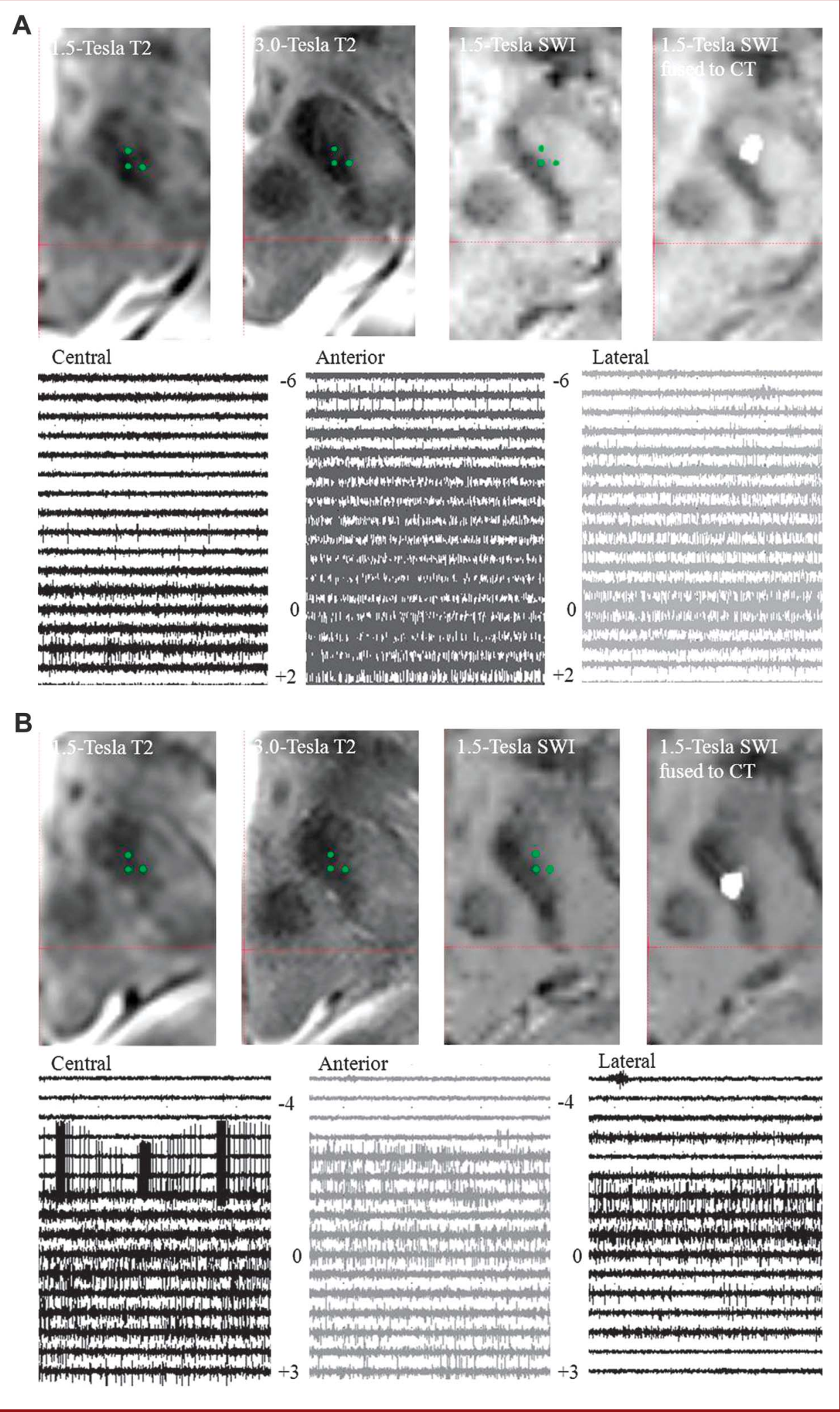




\begin{abstract}
FIGURE 2. Correspondence between microelectrode recordings (MERs) of subthalamic nucleus (STN) neuronal signals and STN representation on 3 different magnetic resonance imaging (MRI) sequences. A, the top left 3 panels show STN representation on axial left-sided midbrain area at target depth in 1 patient on 1.5-T T2, 3.0-T T2, and 1.5-T susceptibility-weighted imaging (SWI) MRI. The green dots represent the central, anterior, and lateral MER channels (both the anterior and lateral channels are located $2 \mathrm{~mm}$ perpendicular to central channel). Stereotactic coordinates of the central dot were $10.5 \mathrm{~mm}$ lateral, $2.0 \mathrm{~mm}$ posterior, and $4.0 \mathrm{~mm}$ ventral relative to the midcommissural point. The top right panel shows postoperative computed tomography (CT) coregistered to 1.5-T SWI MRI. The hyperdense round dot represents the artifact of the electrode as it appears on CT. The bottom 3 panels show corresponding MERs measured for the central, anterior, and lateral microelectrodes. MER started at the top $(6.0 \mathrm{~mm}$ to target) and was advanced in $0.5-\mathrm{mm}$ steps. Each line of recordings represents a single depth. Target depth is represented at 0 . MER were extended $3 \mathrm{~mm}$ after the target depth $(+3$, bottom line of electrophysiological activity). Central, anterior, and lateral channels showed typical electrophysiological STN activity on target depth and were inside STN representation on 1.5- and 3-T T2 MRI. On SWI, however, both the anterior and lateral channels were outside STN representation, indicating a more medial STN representation on SWI compared with both T2 sequences. On the basis of test stimulation, the anterior channel was chosen for definitive DBS lead placement. Stereotactic coordinates (on target depth) of the definitive electrode on CT imaging were $11.2 \mathrm{~mm}$ lateral, $0.2 \mathrm{~mm}$ posterior, and $4.0 \mathrm{~mm}$ ventral relative to the midcommissural point, confirming that the anterior channel was used. Localization of electrode artifact is outside STN representation on SWI, corresponding to anterior microelectrode localization and again confirming the incorrect lateral STN representation on this sequence. B, a comparable design in another patient. Stereotactic coordinates of the central dot were $10.3 \mathrm{~mm}$ lateral, $2.7 \mathrm{~mm}$ posterior, and $4.0 \mathrm{~mm}$ ventral relative to the midcommissural point. All 3 MER channels showed typical electrophysiological STN activity and were inside STN representation on 1.5- and 3-T T2 MRI. On SWI, both the anterior and lateral channels again were outside STN representation. On the basis of test stimulation, the central channel was chosen for definitive DBS lead placement. Stereotactic coordinates of the final electrode on CT imaging were $10 \mathrm{~mm}$ lateral, $4 \mathrm{~mm}$ posterior, and 4.0 $\mathrm{mm}$ ventral relative to the midcommissural point, confirming that the central channel was used. The electrode artifact is inside STN representation on SWI, corresponding to central microelectrode localization.
\end{abstract}

results, however, show that it is of outmost importance to understand the specific characteristics of target nucleus representation on newly implemented MRI sequences in relation to its electrophysiological properties: SWI does not offer an advantage but a disadvantage in delineation of lateral STN borders compared with T2. Thus, conventional T2 sequences remain indispensable for inspection of lateral STN borders.

\section{MER or No MER?}

Recent literature has suggested omitting MER and relying solely on MRI for the determination of optimal lead placement in the STN. ${ }^{5,18,31}$ Comparison between DBS lead placement with the use of MER and placement based solely on 1.5-T T2-weighted MRI sequences showed comparable results. ${ }^{18}$ A possible advantage of MRI-guided DBS is the possibility of performing surgery under general anesthesia. This could offer a major advantage for patient comfort. However, no therapeutic windows between the (rapidly induced) positive and negative effects of stimulation can be determined during surgery owing to a loss of test stimulation. In the present study, the MRI target was used for final lead placement in only $47 \%$ of the placements, whereas the combination of MER and test stimulation determined the final target in the other $53 \%$. It is possible that the development of DBS leads that allow directional stimulation (steering of current), together with intraoperative confirmation of adequate lead placement through CT or MRI, ${ }^{32-}$ ${ }^{35}$ could make MER and test stimulation obsolete in the near future.

\section{Limitations}

We used MER as a gold standard to compare STN representation on SWI and T2 sequences. This does not mean that the
STN measured by MER is the absolute true representation of this nucleus or that the trajectory with best MER activity is necessarily the trajectory chosen for definitive DBS lead placement. This also depends on the findings resulting from intraoperative test stimulation. In our current practice, however, MER is thought to be important for locating the optimal trajectory; trajectories without typical electrophysiological STN activity will at first not be considered for definitive DBS lead placement.

Determination of electrophysiological activity and MER representation on SWI was not performed under blinded conditions. In addition, determination of MER trajectories in relation to STN borders was done only on axial images and at target depth. It could be stated that preferably all depths with measured electrophysiological activity were compared to obtain a more complete insight into the differences between electrophysiological and MRI borders of the STN. Coronal SWI could have been used to evaluate lateral STN border representation, as well as dorsal and ventral borders. ${ }^{9}$ Moreover, different settings of SWI could have been implemented and evaluated to possibly optimize STN representation. However, using STN representation on 1.5-T axial SWI at target depth makes the outcome of this study readily applicable for future direct planning.

During coregistration, especially between sequences, small image fusion errors may be difficult to detect. To optimize registration accuracy between different sequences, all coregistrations were visually inspected by 2 members of the surgical team, 1 of whom was always a DBS surgeon (R.S. or P.v.d.M.). Structures used for verification included the anterior and posterior commissure, course of blood vessels, ventricles, outlines of the mesencephalon, and gyri and sulci. We found comparable high visual accuracy of coregistration for SWI and T2-weighted imaging. 


\section{CONCLUSION}

This study is the first to compare electrophysiological STN measured by MER with the representation of the nucleus on SWI. It shows that SWI does not correctly display the lateral part of the STN. When aiming to target the superolateral sensorimotor part of the STN during DBS surgery, SWI does not offer an advantage but a disadvantage compared with conventional T2. Future research is needed to determine whether these findings may also apply for high-field SWI.

\section{Disclosures}

The DBS team of the Academic Medical Center received unrestricted research grants from Medtronic and received financial compensation for teaching courses for the European Continue Medical Training program. Dr Bot received travel grants from Medtronic. Dr Contarino received speaking fees from Medtronic and Abbvie. Dr Bour acted as an independent advisor for Sapiens steering brain stimulation. Dr Schuurman acts as an independent advisor for Medtronic, Elekta, and Sapiens Steering. The other authors have no personal, financial, or institutional interest in any of the drugs, materials, or devices described in this article.

\section{REFERENCES}

1. Odekerken VJ, van Laar T, Staal MJ, et al. Subthalamic nucleus versus globus pallidus bilateral deep brain stimulation for advanced Parkinson's disease (NSTAPS study): a randomised controlled trial. Lancet Neurol. 2013;12(1): 37-44.

2. Limousin P, Krack P, Pollak P, et al. Electrical stimulation of the subthalamic nucleus in advanced Parkinson's disease. New Engl J Med. 1998;339(16):1105-1111.

3. Guehl D, Cuny E, Benazzouz A, et al. Side-effects of subthalamic stimulation in Parkinson's disease: clinical evolution and predictive factors. Eur J Neurol. 2006;13 (9):963-971.

4. Richter EO, Hoque T, Halliday W, Lozano AM, Saint-Cyr JA. Determining the position and size of the subthalamic nucleus based on magnetic resonance imaging results in patients with advanced Parkinson disease. J Neurosurg. 2004;100(3): 541-546.

5. Ashkan K, Blomstedt P, Zrinzo L, et al. Variability of the subthalamic nucleus: the case for direct MRI guided targeting. Br J Neurosurg. 2007;21(2):197-200.

6. Massey LA, Miranda MA, Zrinzo L, et al. High resolution MR anatomy of the subthalamic nucleus: imaging at $9.4 \mathrm{~T}$ with histological validation. NeuroImage 2012;59(3):2035-2044

7. Dormont D, Ricciardi KG, Tande D, et al. Is the subthalamic nucleus hypointense on T2-weighted images? A correlation study using MR imaging and stereotactic atlas data. AJNR Am J Neuroradiol. 2004;25(9):1516-1523.

8. Hamani C, Richter EO, Andrade-Souza Y, Hutchison W, Saint-Cyr JA, Lozano AM. Correspondence of microelectrode mapping with magnetic resonance imaging for subthalamic nucleus procedures. Surg Neurol. 2005;63(3):249-253; discussion 253

9. Vertinsky AT, Coenen VA, Lang DJ, et al. Localization of the subthalamic nucleus: optimization with susceptibility-weighted phase MR imaging. AJNR Am J Neuroradiol. 2009;30(9):1717-1724.

10. O'Gorman RL, Shmueli K, Ashkan K, et al. Optimal MRI methods for direct stereotactic targeting of the subthalamic nucleus and globus pallidus. Eur Radiol. 2011;21(1):130-136.

11. Ben-Haim S, Asaad WF, Gale JT, Eskandar EN. Risk factors for hemorrhage during microelectrode-guided deep brain stimulation and the introduction of an improved microelectrode design. Neurosurgery. 2009;64(4):754-762; discussion 762-763.

12. Beriault S, Sadikot AF, Alsubaie F, Drouin S, Collins DL, Pike GB. Neuronavigation using susceptibility-weighted venography: application to deep brain stimulation and comparison with gadolinium contrast. J Neurosurg. 2014;121(1) 131-141.

13. Gross RE, Krack P, Rodriguez-Oroz MC, Rezai AR, Benabid AL. Electrophysiological mapping for the implantation of deep brain stimulators for Parkinson's disease and tremor. Mov Disord. 2006;21(suppl 14):S259-S283.
14. Machado A, Rezai AR, Kopell BH, Gross RE, Sharan AD, Benabid AL. Deep brain stimulation for Parkinson's disease: surgical technique and perioperative management. Mov Disord. 2006;21(suppl 14):S247-S258.

15. Hertel F, Zuchner M, Weimar I, et al. Implantation of electrodes for deep brain stimulation of the subthalamic nucleus in advanced Parkinson's disease with the aid of intraoperative microrecording under general anesthesia. Neurosurgery. 2006; 59(5):E1138; discussion E1138.

16. Yamada K, Goto S, Kuratsu J, et al. Stereotactic surgery for subthalamic nucleus stimulation under general anesthesia: a retrospective evaluation of Japanese patients with Parkinson's disease. Parkinsonism Relat Disord. 2007;13(2):101-107.

17. Lefaucheur JP, Gurruchaga JM, Pollin B, et al. Outcome of bilateral subthalamic nucleus stimulation in the treatment of Parkinson's disease: correlation with intraoperative multi-unit recordings but not with the type of anaesthesia. Eur Neurol. 2008;60(4):186-199.

18. Nakajima T, Zrinzo L, Foltynie T, et al. MRI-guided subthalamic nucleus deep brain stimulation without microelectrode recording: can we dispense with surgery under local anaesthesia? Stereotact Funct Neurosurg. 2011;89(5):318-325.

19. Bour LJ, Contarino MF, Foncke EM, et al. Long-term experience with intraoperative microrecording during DBS neurosurgery in STN and GPi. Acta Neurochir (Wien). 2010;152(12):2069-2077.

20. Parent A, Hazrati LN. Functional anatomy of the basal ganglia, II: the place of subthalamic nucleus and external pallidum in basal ganglia circuitry. Brain Res Brain Res Rev. 1995;20(1):128-154.

21. Coenen VA, Prescher A, Schmidt T, Picozzi P, Gielen FL. What is dorso-lateral in the subthalamic nucleus (STN)? A topographic and anatomical consideration on the ambiguous description of today's primary target for deep brain stimulation (DBS) surgery. Acta Neurochirur (Wien). 2008;150(11):1163-1165; discussion 1165.

22. Rodriguez-Oroz MC, Rodriguez M, Guridi J, et al. The subthalamic nucleus in Parkinson's disease: somatotopic organization and physiological characteristics. Brain. 2001;124(pt 9):1777-1790.

23. Kocabicak E, Aygun D, Ozaydin I, et al. Does probe's eye subthalamic nucleus length on T2W MRI correspond with microelectrode recording in patients with deep brain stimulation for advanced Parkinson's disease? Turk Neurosurg. 2013;23(5):658-665.

24. Patil PG, Conrad EC, Aldridge JW, Chenevert TL, Chou KL. The anatomical and electrophysiological subthalamic nucleus visualized by 3-T magnetic resonance imaging. Neurosurgery. 2012;71(6):1089-1095; discussion 1095.

25. Schlaier JR, Habermeyer C, Warnat J, et al. Discrepancies between the MRI- and the electrophysiologically defined subthalamic nucleus. Acta Neurochirur (Wien). 2011;153(12):2307-2318.

26. Weise LM, Seifried C, Eibach S, et al. Correlation of active contact positions with the electrophysiological and anatomical subdivisions of the subthalamic nucleus in deep brain stimulation. Stereotact Funct Neurosurg. 2013;91(5):298-305.

27. Nowinski WL, Thirunavuukarasuu A, Liu J, Benabid AL. Correlation between the anatomical and functional human subthalamic nucleus. Stereotact Funct Neurosurg. 2007;85(2-3):88-93

28. Lefranc M, Derrey S, Merle P, et al. High-resolution 3-dimensional T2*-weighted angiography (HR 3-D SWAN): an optimized 3-T magnetic resonance imaging sequence for targeting the subthalamic nucleus. Neurosurgery. 2014;74(6):615626; discussion 627.

29. Xiao Y, Beriault S, Pike GB, Collins DL. Multicontrast multiecho FLASH MRI for targeting the subthalamic nucleus. Magn Reson Imaging. 2012;30(5):627-640.

30. Elolf E, Bockermann V, Gringel T, Knauth M, Dechent P, Helms G. Improved visibility of the subthalamic nucleus on high-resolution stereotactic MR imaging by added susceptibility $\left(\mathrm{T} 2^{*}\right)$ contrast using multiple gradient echoes. AJNR Am J Neuroradiol. 2007;28(6):1093-1094.

31. Foltynie T, Zrinzo L, Martinez-Torres I, et al. MRI-guided STN DBS in Parkinson's disease without microelectrode recording: efficacy and safety. J Neurol Neurosurg Psychiatry. 2011;82(4):358-363.

32. Pollo C, Kaelin-Lang A, Oertel MF, et al. Directional deep brain stimulation: an intraoperative double-blind pilot study. Brain. 2014;137(pt 7):2015-2026.

33. Ostrem JL, Galifianakis NB, Markun LC, et al. Clinical outcomes of PD patients having bilateral STN DBS using high-field interventional MR-imaging for lead placement. Clin Neurol Neurosurg. 2013;115(6):708-712.

34. Shahlaie K, Larson PS, Starr PA. Intraoperative computed tomography for deep brain stimulation surgery: technique and accuracy assessment. Neurosurgery. 2011; 68(1 suppl operative):114-124; discussion 124 .

35. Contarino MF, Bour LJ, Verhagen R, et al. Directional steering: a novel approach to deep brain stimulation. Neurology. 2014;83(13):1163-1169. 


\section{COMMENTS}

T his article examines concordance between electrophysiological recordings from the subthalamic nucleus (STN) and different magnetic resonance imaging (MRI) sequences. The authors conclude that T2-weighted imaging provides better concordance with microelectrode recordings (MER) than do susceptibility-weighted imaging (SWI). These findings are of potential practical importance by limiting the number of sequential brain passes required to accurately target the STN. However, there are a number of limitations that should be considered.

MRI distortion is a well-known phenomenon that can limit targeting accuracy in functional neurosurgery. ${ }^{1}$ This can often be addressed by using distortion correction algorithms, but these were not mentioned in this article.

The authors used visual inspection to determine the accuracy of coregistration. However, image coregistration can introduce significant errors that are not always readily detectable. ${ }^{2}$ In our practice, using commercially available planning software to coregister different stereotactic MRI sequences appears accurate when the concordance of brain structures is being examined. However, the fiducial markers of the different stereotactic sequences do not always superimpose perfectly. This error may go undetected when nonstereotactic postoperative CT images are coregistered with stereotactic MRI, as performed in this study.

MER activity was situated consistently inside hypointense STN contour representation in $99 \%$ of 0.5 -T T2-weighted images and $100 \%$ of 3-T T2-weighted images. This supports the suggestion that stereotactic MRI confirming lead placement within the T2-weighted STN can replace MER as a method of lead verification. ${ }^{3}$ However, the authors used the MRI target in only $47 \%$ of lead placements. This discrepancy suggests that more precise targeting within the visible STN on T2-weighted imaging and further reduction in stereotactic targeting errors are required to improve the accuracy of first-pass lead placement. ${ }^{4}$

Although this article does not present any clinical data, it is clearly of practical value to the authors and may influence which MRI sequences neurosurgeons use when performing STN DBS. Functional neurosurgeons should certainly be encouraged to audit the techniques they use and eventually to examine their impact on clinical outcome.

Ludvic Zrinzo London, United Kingdom

1. Sankar T, Lozano AM. Magnetic resonance imaging distortion in functional neurosurgery. WNEU. 2011;75:29-31.

2. O'Gorman RL, Jarosz JM, Samuel M, et al. CT/MR image fusion in the postoperative assessment of electrodes implanted for deep brain stimulation. Stereotact Funct Neurosurg. 2009;87:205-210.
3. Zrinzo L, Foltynie T, Limousin P, et al. Image-guided and image-verified deep brain stimulation. Mov Disord. 2013;28:254.

4. Holl EM, Petersen EA, Foltynie T, et al. Improving targeting in image-guided frame-based deep brain stimulation. Neurosurgery. 2010;67:ons437-ons447.

$\mathrm{n}$ this article, the authors compare the susceptibility-weighted imaging (SWI) magnetic resonance imaging (MRI) sequence with the T2 sequence and the "gold standard" microelectrode recording (MER) broader definition demonstrating that SWI sequences are still not as good as MER in defining the target structure borders. As imaging becomes used more frequently in the placement of deep brain stimulation leads, investigations of this kind are necessary. As the authors mention, that there are advantages in doing these procedures with MRI guidance alone such as general anesthesia. At the present time and given the ease and benefits of MER-based localization, which include (1) direct patient feedback; (2) direct knowledge the functional location within the target nuclei-ie the sensorimotor areas (which at the present is believed to be the optimal location to place the lead); (3) lack of information on how well, and even if, steerable leads will improve the ability to program around less than optimally placed leads. MER (or some form of detailed physiologic assessment in the operating room) is still necessary given that it is the only tool that can test the location of the tip at the actual moment it is placed in the target location.

Jay L. Shils

Chicago, Illinois

$\mathbf{T}$ his study examines multielectrode recording (MER) concordance of 1.5-/3-T T2 and 1.5- susceptibility-weighted imaging (SWI) magnetic resonance imaging (MRI) for subthalamic nucleus (STN) deep brain stimulation surgery. The authors conclude that T2 MRI provides a more accurate and precise MER relative to SWI. In particular, the authors focus on the inaccurate depiction of SWI of the lateral borders of the STN on 1.5-T MRI. As a result, there is a potential for nonoptimal targeting if 1.5-T SWIMRI is used as the primary imaging modality for stereotactic target selection.

The authors demonstrate excellent MER/MRI concordance rates when using T2-weighted imaging ( $>95 \%$ for 1.5 and $3 \mathrm{~T}$ ) for targeting. Of note, the authors did not use high-field strength (3.0 T) SWI MRI in their study. As these high-field-strength MRIs become more routinely used in practice, SWI depiction of the STN will likely improve in tandem. In this article as a whole, the authors lend credence to the concept of using image-based rather than MER-based lead placement. As the quality of MRI scans steadily improves (which will theoretically improve the depiction of the STN on SWI), the need for MER will fade.

Overall, the topic is interesting and the article is well written and provides a useful addition to the body of STN deep brain stimulation surgery literature.

Brian Harris Kopell Jonathan Rasouli New York, New York 